\title{
Does the combination of photobiomodulation therapy (PBMT) and static magnetic fields (sMF) potentiate the effects of aerobic endurance training and decrease the loss of performance during detraining? A randomised, triple- blinded, placebo-controlled trial
}

Paulo Roberto Vicente de Paiva ${ }^{1,2}$, Heliodora Leão Casalechi ${ }^{1}$, Shaiane Silva Tomazoni ${ }^{3,4}$, Caroline dos Santos Monteiro Machado ${ }^{1,2}$, Neide Firmo Ribeiro ${ }^{1,2}$, Amanda Lima Pereira', Marcelo Ferreira Duarte de Oliveira ${ }^{1,2}$, Marjury Nunes da Silva Alves ${ }^{1}$, Maiara Conceição dos Santos ${ }^{1}$, Inti Ernesto Torrico Takara ${ }^{1}$, Eduardo Foschini Miranda', Paulo de Tarso Camillo de Carvalho ${ }^{2}$ and Ernesto Cesar Pinto Leal-Junior $r^{1,2,3,4^{*}}$

\footnotetext{
Abstract

Background: Photobiomodulation (PBMT) is a therapy that uses non-ionising forms of light, including low-level lasers and light-emitting diodes (LEDs) that may be capable of modulating cellular activity. Some biological processes may also interact with static magnetic fields (sMF), leading to modulatory effects on cells. Previous studies have verified that the combination of PBMT and SMF (PBMT/SMF) enhances the performance of individuals during aerobic training programs. The detraining period can cause losses in aerobic capacity. However, there is no evidence of the existence of any recourse that can decrease the effects of detraining. We aimed to investigate the effects of PBMT/sMF application during training and detraining to assess the effectiveness of this treatment in reducing the effects of detraining.

\footnotetext{
* Correspondence: ernesto.leal.junior@gmail.com

${ }^{1}$ Laboratory of Phototherapy and Innovative Technologies in Health (LaPIT), Nove de Julho University, Rua Vergueiro, 235/249, São Paulo, SP 01504-001, Brazil

${ }^{2}$ Postgraduate Program in Rehabilitation Sciences, Nove de Julho University, São Paulo, SP, Brazil

Full list of author information is available at the end of the article
}

(c) The Author(s). 2020 Open Access This article is licensed under a Creative Commons Attribution 4.0 International License, which permits use, sharing, adaptation, distribution and reproduction in any medium or format, as long as you give appropriate credit to the original author(s) and the source, provide a link to the Creative Commons licence, and indicate if changes were made. The images or other third party material in this article are included in the article's Creative Commons licence, unless indicated otherwise in a credit line to the material. If material is not included in the article's Creative Commons licence and your intended use is not permitted by statutory regulation or exceeds the permitted use, you will need to obtain permission directly from the copyright holder. To view a copy of this licence, visit http://creativecommons.org/licenses/by/4.0/. The Creative Commons Public Domain Dedication waiver (http://creativecommons.org/publicdomain/zero/1.0/) applies to the data made available in this article, unless otherwise stated in a credit line to the data. 
(Continued from previous page)

Methods: Sixty male volunteers were randomly allocated into four groups - participants who received PBMT/sMF during the training and detraining (PBMT/SMF + PBMT/SMF); participants who received PBMT/sMF during the training and a placebo in the detraining (PBMT/SMF + Placebo); participants who received a placebo during the training and PBMT/SMF in the detraining (Placebo+PBMT/SMF); and participants who received a placebo during the training and detraining (Placebo+Placebo). Participants performed treadmill training over 12 weeks (3 sessions/ week), followed by 4 weeks of detraining. PBMT/sMF was applied using a 12-diode emitter (four 905 nm superpulsed lasers, four $875 \mathrm{~nm}$ light-emitting diodes (LEDs), four $640 \mathrm{~nm}$ LEDs, and a $35 \mathrm{mT}$ magnetic field) at 17 sites on each lower limb (dosage: $30 \mathrm{~J}$ per site). The data were analysed by two-way repeated measures analysis of variance (ANOVA, time vs experimental group) with post-hoc Bonferroni correction.

Results: The percentage of change in time until exhaustion and in maximum oxygen consumption was higher in the PBMT/sMF + PBMT/sMF group than in the Placebo+Placebo group at all time-points $(p<0.05)$. Moreover, the percentage of decrease in body fat at the 16th week was higher in the PBMT/sMF + PBMT/sMF group than in the Placebo+Placebo group ( $p<0.05)$.

Conclusions: PBMT/SMF can potentiate the effects of aerobic endurance training and decrease performance loss after a 4-week detraining period. Thus, it may prove to be an important tool for both amateur and highperformance athletes as well as people undergoing rehabilitation.

Trial registration: NCT03879226. Trial registered on 18 March 2019.

Keywords: Low-level laser therapy, Light-emitting diode therapy, Phototherapy, Endurance exercise, Deconditioning

\section{Background}

Regular physical activity is recommended for improving general health, performance enhancement, and the rehabilitation of chronic diseases [1, 2]. In addition, aerobic exercise improves cardiovascular health and decreases body fat mass [3-7]. However, individuals often discontinue exercise in response to illness, injury, or other factors that may alter their capacity for physical activity, causing a rapid loss of aerobic conditioning that can be observed after 2-4 weeks [8, 9]. Currently, there is insufficient evidence to support the ability of any particular resource or method to decrease the effects of detraining.

Photobiomodulation therapy (PBMT) is a therapy that uses non-ionising light sources, such as lasers, light-emitting diodes (LEDs), and broadband light, from the visible to the infrared spectrum [10]. PBMT elicits a nonthermal process where light interacts with chromophores leading to photophysical and photochemical reactions in different tissues, thereby promoting cell metabolism modulation [11, 12]. PBMT has been investigated for promoting exercise-related ergogenic effects [10]. Leal-Junior et al. (2009), Antonialli et al. (2014), and de Paiva et al. (2016) observed that the use of PBMT with exercise can reduce creatine kinase (CK) activity through its protective effects on skeletal muscle tissue, allowing for a faster recovery [13-15]. Other studies indicate that PBMT can decrease blood lactate levels and thus, help to improve performance during exercise [16-18].

Static magnetic fields (sMF) are force fields that are produced by moving electrical currents that act on other mobile charges, which can interact with several biological processes $[19,20]$, also leading to the modulation of cellular metabolism [20-22]. Previous studies have reported that the use of sMF in association with PBMT generates greater effects in cellular metabolism than the use of PBMT alone [23]. In the clinical context, it has been demonstrated that the association of PBMT with sMF (PBMT/sMF) promotes ergogenic effects [18, 24]. Moreover, Miranda et al. (2018) verified that this combination enhances the performance of individuals during an aerobic training program, increasing the percentage of change of maximum oxygen consumption $\left(\mathrm{VO}_{2} \mathrm{max}\right)$ and time until exhaustion after 12 weeks of the training protocol [25].

To the best of our knowledge, no previous study has investigated the effects of PBMT/sMF in the detraining or deconditioning period. However, the effects of PBMT on the oxidative metabolism of peripheral blood cells (erythrocytes, granulocytes, and lymphocytes), leading to an enhanced oxygen-carrying ability of blood, was previously demonstrated in heparinised blood samples [26]. According to Wasik et al., the partial pressure of oxygen $\left(\mathrm{PO}_{2}\right)$ and oxygen saturation $\left(\mathrm{SaO}_{2}\right)$ increases after PBMT irradiation [26]. These findings suggest that PBMT could attenuate the 
loss of performance observed during the detraining period after an aerobic training program through increased oxygen transportation, and consequently, the ability of muscles to use it, which is decreased during the detraining period.

Therefore, PBMT/sMF may prove to be an important tool for both amateur and high-performance athletes as well as for people in the process of rehabilitation who discontinue exercise due to illness, injury, or other factors. With this perspective, we aimed to investigate the effects of PBMT/sMF during the training and detraining period in maintaining the benefits acquired in an aerobic training program.

\section{Methods}

The protocol and methods used in this study were previously published in a peer-reviewed scientific journal [27].

\section{Design and ethical aspects}

A randomised, triple-blind (volunteers, therapists, and assessors), placebo-controlled clinical trial was performed at the Laboratory of Phototherapy and Innovative Technologies in Health (LaPIT). The study followed the ethical guidelines of and was approved by the Research Ethics Committee of Nove de Julho University (protocol number 1781602). The protocol was prospectively registered at ClinicalTrials.org (NCT03879226) by 18 March 2019. The first volunteer was enrolled at 25 March 2019, and all volunteers signed an informed consent form at the time of enrolment in the study.

\section{Subjects and sample size}

As no previous studies have assessed the effects of $\mathrm{PBMT} / \mathrm{sMF}$ during the detraining period after an aerobic training program, the number of participants per group in the present study was calculated based on a pilot study, with five volunteers per group, conducted by our research group to estimate the sample size. A beta value of $20 \%$ and alpha value of $5 \%$ were used to calculate the sample size.

The pilot study showed that applying PBMT/sMF during the detraining period resulted in a time to exhaustion (the primary outcome of this study) of $923.60 \mathrm{~s}$ (standard deviation, $65.77 \mathrm{~s}$ ) during the progressive treadmill test; whereas applying the placebo during the detraining period resulted in a time to exhaustion of $846.82 \mathrm{~s}$ (standard deviation, $99.23 \mathrm{~s}$ ). We used the Researcher's Toolkit to calculate the sample (https://www.dssresearch.com/KnowledgeCenter/toolkitcalculators/samplesizecalculators.aspx).

Based on the parameters used to calculate the sample, we determined that 15 volunteers per group, for a total of 60 volunteers, was appropriate. Therefore, predicting a $20 \%$ sample loss, up to 72 healthy and physically inactive male volunteers aged $18-35$ years who were students at the Nove de Julho University, would be recruited for the study to ensure a final sample size of 60 volunteers. As the PBMT/sMF device used in the study has no harmful thermal effects, volunteers of all ethnicities were recruited [28].

\section{Patient and public involvement statement}

Patients and/or the public were not involved in the design, recruitment to, and conduct of this study. At the end of the study, the main results were disseminated to participants by email.

\section{Eligibility criteria \\ Inclusion criteria}

Healthy men aged 18-35 years from all ethnicities, who were non-smokers, with no history of a musculoskeletal injury in the hip and knee regions in the 2 months before the study, who did not regularly use pharmacological agents and/or nutritional supplements, and who completed at least $80 \%$ of the study procedures, were included in the study.

\section{Exclusion criteria}

Volunteers who showed any musculoskeletal injury in the 2 months before the study, were injured during the study, regularly used any type of nutritional supplement or pharmacological agent, or who showed signs and symptoms of any neurological, metabolic, inflammatory, pulmonary, oncological, or cardiovascular disease that may limit the execution of high-intensity exercises, were excluded from the study.

\section{Randomisation and blinding and experimental groups}

In order to avoid selection bias, and to ensure that all individuals were randomly allocated to any group, balanced block randomisation was performed based on the primary outcome (time to exhaustion in the progressive treadmill test) by a researcher who had no contact with the study subjects or the other researchers involved in the project.

A researcher programmed the device (PBMT/sMF or placebo) and was instructed not to inform the volunteers or other researchers of the type of treatment (PBMT/ sMF or placebo). The sounds and signals emitted from the device as well as the information displayed on the screen were identical, regardless of the type of treatment (PBMT/sMF or placebo), providing the appropriate blinding of volunteers and therapists. All volunteers used opaque glasses during the treatments both for safety and to aid in blinding. Thus, volunteers, evaluators, and therapists were blinded to maintain the triple-blind design.

Randomisation labels were created through the random.org website, and a series of sealed, opaque, and 
numbered envelopes were used to ensure confidentiality and to determine to which experimental group each volunteer was to be allocated. Volunteers were allocated as described below:

PBMT/sMF + PBMT/sMF: PBMT/sMF before and after the aerobic training sessions (12 weeks, 3 times a week) and PBMT/sMF during the detraining period (4 weeks, 3 times a week).

PBMT/sMF + Placebo: PBMT/sMF before and after the aerobic training sessions (12 weeks, 3 times a week) and placebo during the detraining period (4 weeks, 3 times a week).

Placebo+PBMT/sMF: Placebo before and after the aerobic training sessions (12 weeks, 3 times a week) and $\mathrm{PBMT} / \mathrm{sMF}$ during the detraining period (4 weeks, 3 times a week).

Placebo+Placebo: Placebo before and after the aerobic training sessions (12 weeks, 3 times a week) and placebo during the detraining period (4 weeks, 3 times a week).

The individuals were subjected to 12 consecutive weeks of aerobic endurance training on a motorised treadmill, with 3 training sessions per week on nonconsecutive days.

After the 12-week training period, the volunteers received the treatment (either PBMT/sMF or placebo, depending on the group to which they were allocated) during the 4 weeks ( 3 times a week) without training.

The evaluations described below were performed before starting the protocol (baseline) and after 4, 8, and
12 weeks of aerobic endurance training as well as after 4 weeks without training (detraining period) at the 16th week. A flowchart summarising the procedures of this study is presented in Fig. 1.

\section{Procedures \\ Cardiorespiratory evaluation}

Ergospirometry is currently the most accurate cardiorespiratory fitness test [29]. In this study, we used a progressive treadmill protocol previously used by our research group [25, 30-32]. An ergometric treadmill model Super ATL AQ5, and a gas analyser model VO2000, which was connected to a microcomputer for data visualisation and recording were used. The ergospirometry test started with the treadmill set at a $1 \%$ slope, with an initial speed of $3 \mathrm{~km} / \mathrm{h}$, which was maintained until the end of the 3-min warm-up phase. After the warm-up phase, the speed was increased by $1 \mathrm{~km} / \mathrm{h}$ every minute until a maximum speed of $16 \mathrm{~km} / \mathrm{h}$ was reached. The end of the test was defined by the volunteers, who were instructed to perform the test until they reached exhaustion [25, 30-32]. The recovery phase then began, lasting $3 \mathrm{~min}$ at a speed of $6 \mathrm{~km} / \mathrm{h}$. During the test, data on the total time of the exercise (time to exhaustion), maximum oxygen uptake in absolute and relative values in relation to body mass $\left(\mathrm{VO}_{2 \max }\right)$ were recorded [25, 30-32]. The chosen parameters represent those that are most commonly used for this purpose,

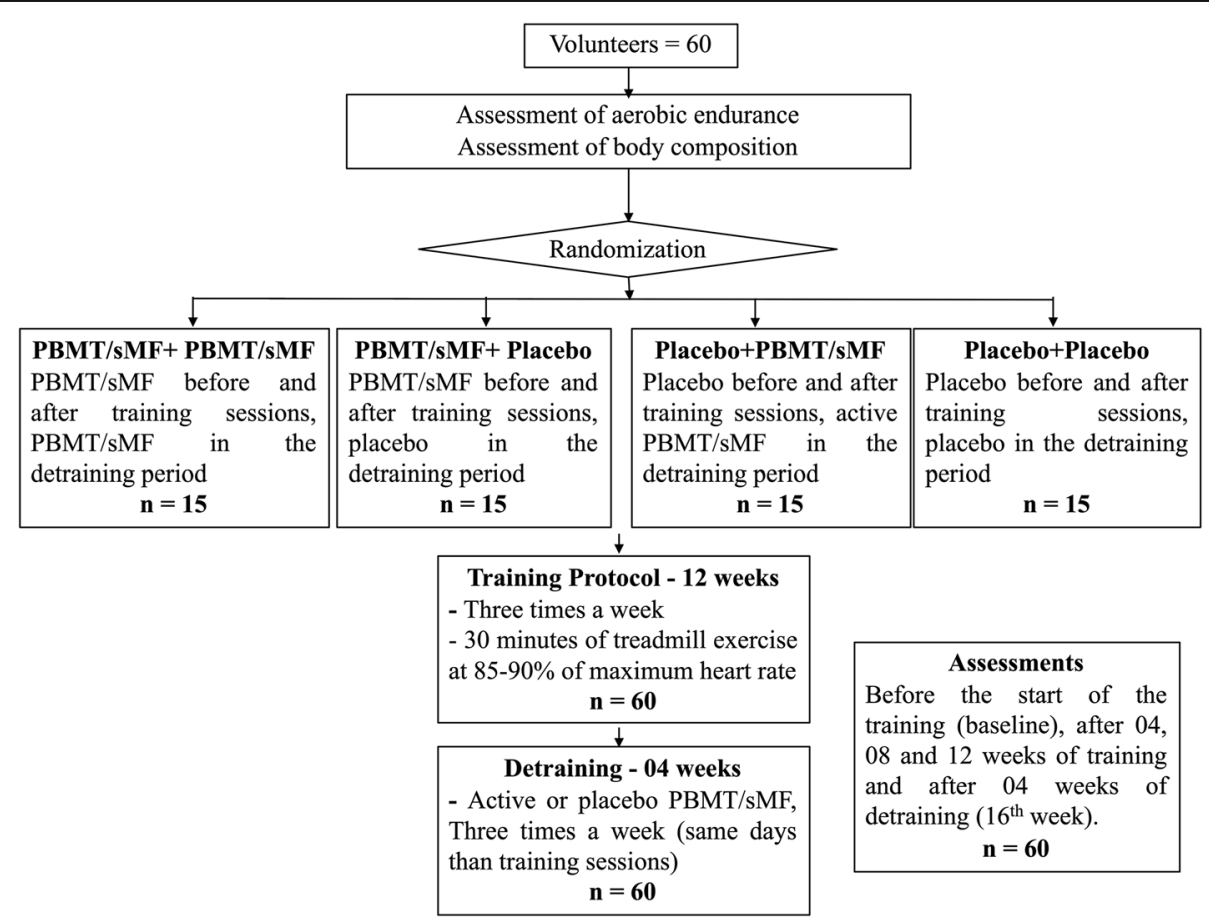

Fig. 1 CONSORT flowchart 
and as such, these data were used to assess the performance of the subjects in the exercise protocol [29].

The entire test was monitored by electrocardiography and blood pressure measurements. If any abnormal changes in heart rate or blood pressure were found, or if the volunteer had any complaint, the test was discontinued, and the volunteer was excluded from the study.

\section{Body composition evaluation}

All body composition evaluations were performed by the same technician (level II of the International Society for the Advancement of Kineanthropometry - ISAK), using the procedures established by the ISAK [33]. The height, body mass, length of body segments, diameters, perimeters, and skinfolds of the participants were measured to assess the muscle mass, adipose mass, residual mass, bone mass, and epithelial mass [25].

\section{Aerobic training}

Aerobic training was performed on a treadmill, with and without PBMT/sMF, 3 times a week on non-consecutive days, for 12 weeks. Each training session was supervised by a certified trainer and lasted $30 \mathrm{~min}$. The speed of the motorised treadmill was tailored to each participant for every training session. The speed of the treadmill was varied during the exercise to keep the heart rate of the volunteers between 85 to $90 \%$ of maximum heart rate [25]; the heart rate of the volunteers was monitored during the entire exercise session using a heart rate monitor. The maximum heart rate was determined during the cardiorespiratory evaluation protocol described above $[25,31]$.

The protocol was interrupted when criteria established by the American Heart Association guidelines were met. The subjects were also evaluated using the 0-10 Borg scale, which is a simple method for classifying perceived exertion, feelings of physical fatigue, or dyspnoea.

\section{Photobiomodulation therapy (PBMT) and static magnetic fields (sMF)}

PBMT/sMF or placebo were applied before and after each training session, as aforementioned. This irradiation protocol was tested and optimised in a previous study conducted by our research group [25]. The results of this study showed that PBMT/sMF before and after each training session was the most effective in enhancing the effects of aerobic training [25]. PBMT/sMF was applied bilaterally using the direct contact method with light pressure on the skin at different sites, namely nine sites on the knee extensor muscles (Fig. 2a), six sites on the knee flexor muscles, and two sites on the plantar flexor muscles (Fig. 2b).

To apply PBMT/sMF, a 12-diode cluster was used, including four $905 \mathrm{~nm}$ laser diodes $(12.5 \mathrm{~W}$ peak power of

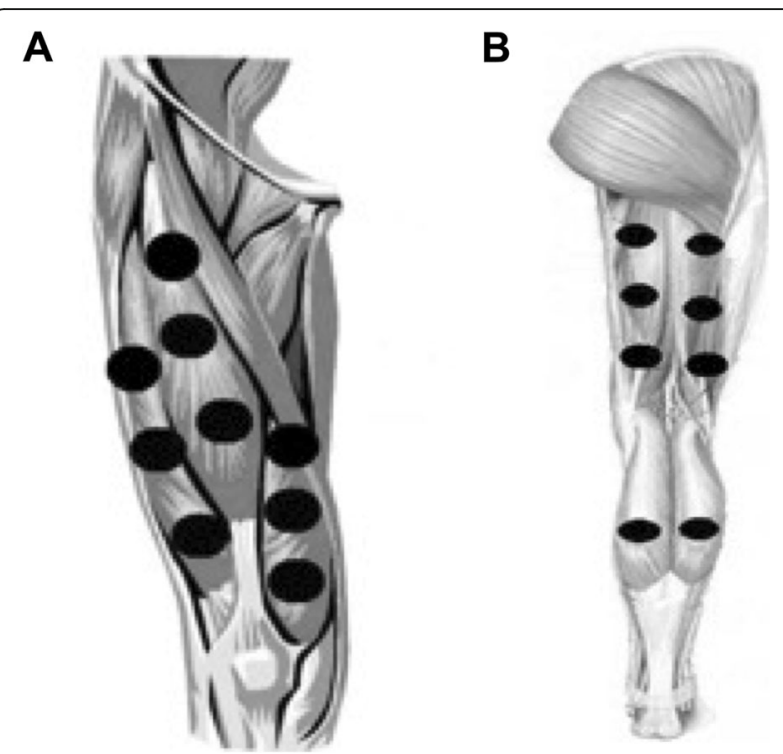

Fig. 2 a: Treatment sites at knee extensor muscles $\mathbf{b}$ : Treatment sites at knee-flexor and ankle plantar-flexor muscles

each diode, $250 \mathrm{~Hz}$ ), four $875 \mathrm{~nm}$ LEDs (average power of each diode, $17.5 \mathrm{~mW}$ ), four $640 \mathrm{~nm}$ LEDs (average power of each diode, $15 \mathrm{~mW}$ ), and a static magnetic field (35 mT) manufactured by Multi Radiance Medical (Solon, OH - USA). Considering the large irradiation area used in the present project, the use of diode clusters was essential for the application of PBMT/sMF; the cluster was circular and had a total irradiation area of 20 $\mathrm{cm}^{2}$.

The dose for active PBMT/sMF was $30 \mathrm{~J}$ per area (228 $\mathrm{s}$ of irradiation in each area) [14], $510 \mathrm{~J}$ of irradiated energy per lower limb $[18,25,31]$, and $1020 \mathrm{~J}$ of total irradiated energy $[18,25,31]$. The dose used at each site was previously tested and optimised by our research group using the same PBMT/sMF device and demonstrated favourable results in terms of enhancing performance and muscle recovery [14]. Furthermore, the irradiation sites were also previously optimised by our research group $[18,25,31]$. The complete description of $\mathrm{PBMT} / \mathrm{sMF}$ parameters is presented in Table 1.

\section{Statistical analysis}

The primary outcome of this study was time until exhaustion, obtained from the progressive treadmill test. The secondary outcomes were $\mathrm{VO}_{2} \mathrm{max}$ in relation to body mass and body fat percentage. The intention-totreat analysis was followed a priori, and all data were analysed by a blinded researcher who was not involved in the data collection. The findings were tested for normality using the Shapiro-Wilk test and were determined to have a normal distribution. Data were expressed as the mean and standard deviation and were analysed by 
Table 1 Parameters for PBMT/sMF

\begin{tabular}{|c|c|}
\hline Number of lasers & 4 Super-pulsed (infrared) \\
\hline Wavelength (nm) & $905( \pm 1)$ \\
\hline Frequency $(\mathrm{Hz})$ & 250 \\
\hline Peak power $(W)$ - each & 12.5 \\
\hline Average mean optical output (mW) - each & 0.3125 \\
\hline Power density $\left(\mathrm{mW} / \mathrm{cm}^{2}\right)$ - each & 0.71 \\
\hline Energy density $\left(\mathrm{J} / \mathrm{cm}^{2}\right)$ - each & 0.162 \\
\hline Dose $(J)$ - each & 0.07125 \\
\hline Spot size of laser $\left(\mathrm{cm}^{2}\right)$ - each & 0.44 \\
\hline Number of red LEDs & 4 Red \\
\hline Wavelength of red LEDs (nm) & $640( \pm 10)$ \\
\hline Frequency $(\mathrm{Hz})$ & 2 \\
\hline Average optical output (mW) - each & 15 \\
\hline Power density $\left(\mathrm{mW} / \mathrm{cm}^{2}\right)$ - each & 16.66 \\
\hline Energy density $\left(J / \mathrm{cm}^{2}\right)$ - each & 3.8 \\
\hline Dose $(J)$ - each & 3.42 \\
\hline Spot size of red LED $\left(\mathrm{cm}^{2}\right)$ - each & 0.9 \\
\hline Number of infrared LEDs & 4 Infrared \\
\hline Wavelength of infrared LEDs (nm) & $875( \pm 10)$ \\
\hline Frequency $(\mathrm{Hz})$ & 16 \\
\hline Average optical output (mW) - each & 17.5 \\
\hline Power density $\left(\mathrm{mW} / \mathrm{cm}^{2}\right)$ - each & 19.44 \\
\hline Energy density $\left(\mathrm{J} / \mathrm{cm}^{2}\right)$ - each & 4.43 \\
\hline Dose $(J)$ - each & 3.99 \\
\hline Spot size of LED $\left(\mathrm{cm}^{2}\right)$ - each & 0.9 \\
\hline Magnetic Field (mT) & 35 \\
\hline Irradiation time per site (sec) & 228 \\
\hline Total dose per site $(J)$ & 30 \\
\hline Total dose applied per lower limb (J) & 510 \\
\hline Aperture of device $\left(\mathrm{cm}^{2}\right)$ & 20 \\
\hline Application mode & Cluster probe held stationary in skin contact with a 90-degree angle and slight pressure \\
\hline
\end{tabular}

Table 2 Participants' characteristics in absolute values

\begin{tabular}{lllll}
\hline & PBMT/sMF + PBMT/sMF & PBMT/sMF + Placebo & Placebo + PBMT/sMF & Placebo + Placebo \\
\hline Age (years) & $24.79 \pm 5.22$ & $23.82 \pm 4.29$ & $23.81 \pm 6.01$ & $28.83 \pm 5.52$ \\
Body mass (kg) & $78.09 \pm 16.49$ & $73.71 \pm 14.33$ & $71.24 \pm 13.50$ & $79.99 \pm 12.12$ \\
Height (cm) & $174.71 \pm 7.32$ & $175.94 \pm 4.94$ & $173.88 \pm 6.67$ & $173.17 \pm 7.59$ \\
Body mass index & $25.55 \pm 5.16$ & $23.78 \pm 4.43$ & $23.52 \pm 4.10$ & $26.62 \pm 3.17$ \\
Heart rate (beats per minute) & $82.21 \pm 11.34$ & $80.65 \pm 13.93$ & $84.13 \pm 14.12$ & $84.08 \pm 11.14$ \\
Systolic blood pressure (mmHg) & $114.29 \pm 5.56$ & $117.07 \pm 14.04$ & $110.00 \pm 8.94$ & $120.00 \pm 18.59$ \\
Diastolic blood pressure (mmHg) & $80.00 \pm 5.55$ & $81.18 \pm 7.81$ & $77.50 \pm 7.75$ & $85.00 \pm 13.82$ \\
\hline
\end{tabular}

${ }^{a}$ Calculated as $\mathrm{kg} / \mathrm{m}^{2}$; VO2 max: oxygen uptake. Data is expressed in average and standard deviation ( \pm ) 
two-way repeated measures analysis of variance (ANOVA, time vs experimental group) with post-hoc Bonferroni correction. Data were also analysed in terms of the absolute values and the percentage of change based on the values established at baseline. The significance level was set at $p<0.05$. In the graphs, data are expressed as the mean and standard error of the mean (SEM).

\section{Results}

All 60 participants completed the full 16-week study; there were no dropouts and there were no adverse effects reported. The characteristics of the volunteers are summarised in Table 2. Statistical analysis revealed that there were no significant differences $(p>0.05)$ between the volunteers from the four experimental groups with respect to the participants' characteristics.

Table 3 shows the results of the progressive cardiopulmonary test in absolute values for the different variables analysed in all experimental groups of this study. There were no statistically significant differences in time until exhaustion or body fat percentage between the groups. Regarding the $\mathrm{VO}_{2} \mathrm{max}$, all groups treated with PBMT/ sMF showed an increase in $\mathrm{VO}_{2} \max$ over time when compared to the Placebo+Placebo group. This difference was statistically significant in the PBMT/sMF + PBMT/ sMF group $(p<0.05, p<0.05, p<0.05$, and $p<0.01)$ and the PBMT/sMF + Placebo group $(p<0.01, p<0.001, p<$ 0.001 , and $p<0.05$ ) at all experimental time-points (4th, 8 th, 12th, and 16th weeks, respectively).
With respect to the time until exhaustion, Fig. 3 shows the percentage of change in the evaluated time-points. The PBMT/sMF + PBMT/sMF group showed a statistically significant difference $(p<0.05, \quad p<0.0001, \quad p<$ 0.0001 , and $p<0.0001)$ at all experimental time-points (4th, 8th, 12th, and 16th week, respectively) when compared to the Placebo+Placebo group. In the detraining period (16th week), the PBMT/sMF + PBMT/sMF group also showed a statistically significant difference $(p<0.05)$ compared to the PBMT/sMF + Placebo group. Moreover, the group treated with $\mathrm{PBMT} / \mathrm{sMF}$ only in the training period (PBMT/sMF + Placebo) or in the detraining period (Placebo+PBMT/sMF), showed a statistically significant difference $(p<0.01$ and $p<0.05$, respectively) in the percentage of change of time until exhaustion when compared to the placebo.

Figure 4 graphically represents the percentage of change in $\mathrm{VO}_{2}$ max in relation to body mass in the study participants. The PBMT/sMF + PBMT/sMF group showed a statistically significant difference $(p<0.0001$, $p<0.0001, p<0.0001$, and $p<0.0001)$ when compared to the Placebo +Placebo group, at all experimental timepoints (4th, 8th, 12th, and 16th week, respectively). In the 16th week, the PBMT/sMF + PBMT/sMF group also presented a statistically significant difference compared to the Placebo+PBMT/sMF group $(p<0.0001)$ and the PBMT/sMF + Placebo group $(p<0.001)$. The groups that received PBMT/sMF only in the training period (PBMT/ $\mathrm{sMF}+$ Placebo) or in the detraining period (Placebo+PBMT/sMF), also presented a statistically significant

Table 3 Progressive endurance test variables in absolute values

\begin{tabular}{|c|c|c|c|c|c|}
\hline & Baseline & 4 weeks & 8 weeks & 12 weeks & 16 weeks \\
\hline \multicolumn{6}{|l|}{ Time until exhaustion (sec) } \\
\hline PBMT/sMF + PBMT/sMF & $767.29 \pm 77.58$ & $887.86 \pm 81.37$ & $921.21 \pm 101.07$ & $986.86 \pm 125.53$ & $932.57 \pm 110.46$ \\
\hline PBMT/sMF + Placebo & $812.53 \pm 121.46$ & $934.35 \pm 134.16$ & $980.24 \pm 149.28$ & $1025.65 \pm 165.58$ & $915.53 \pm 147.63$ \\
\hline Placebo + PBMT/sMF & $853.54 \pm 146.12$ & $906.5 \pm 167.54$ & $949.25 \pm 176.76$ & $961 \pm 153.46$ & $940.06 \pm 142.37$ \\
\hline Placebo + Placebo & $801.67 \pm 122.49$ & $855.83 \pm 134.66$ & $853 \pm 119.39$ & $892.67 \pm 155.03$ & $823.08 \pm 160.85$ \\
\hline \multicolumn{6}{|l|}{$\mathrm{VO}_{2 \max }(\mathrm{mL} / \mathrm{kg} / \mathrm{min})$} \\
\hline PBMT/sMF + PBMT/sMF & $21.94 \pm 2.71$ & $30.81 \pm 4.38^{*}$ & $30.87 \pm 4.49^{*}$ & $30.64 \pm 4.98^{*}$ & $30.26 \pm 5.42^{* *}$ \\
\hline PBMT/sMF + Placebo & $24.91 \pm 6.08$ & $32.06 \pm 8.53^{* *}$ & $33.48 \pm 7.97^{* * *}$ & $33.34 \pm 9.13^{* * *}$ & $29.46 \pm 8.16^{*}$ \\
\hline Placebo + PBMT/sMF & $24.61 \pm 6.14$ & $26.93 \pm 7.25$ & $28.27 \pm 6.41$ & $27.4 \pm 5.51$ & $28.15 \pm 5.44$ \\
\hline Placebo + Placebo & $22.06 \pm 5.12$ & $24.68 \pm 4.96$ & $24.89 \pm 5.60$ & $24.34 \pm 5.24$ & $22.38 \pm 5.31$ \\
\hline \multicolumn{6}{|l|}{ Fat percentage } \\
\hline PBMT/sMF + PBMT/sMF & $24.55 \pm 10.64$ & $22.61 \pm 9.07$ & $22.3 \pm 8.74$ & $21.59 \pm 9.58$ & $20.84 \pm 8.65$ \\
\hline PBMT/sMF + Placebo & $26.16 \pm 6.46$ & $25.39 \pm 7.31$ & $25.19 \pm 6.7$ & $24.85 \pm 7.21$ & $25.2 \pm 7.98$ \\
\hline Placebo + PBMT/sMF & $18.8 \pm 10.39$ & $17.83 \pm 10.00$ & $17.62 \pm 10.00$ & $16.96 \pm 9.14$ & $17.24 \pm 9.19$ \\
\hline Placebo + Placebo & $21.05 \pm 9.60$ & $20.29 \pm 9.24$ & $19.91 \pm 8.89$ & $19.43 \pm 8.56$ & $19.13 \pm 8.53$ \\
\hline
\end{tabular}

Data are expressed as means and standard deviations $( \pm) . \mathrm{VO}_{2}$ max: maximum oxygen uptake. ${ }^{*}$ indicates a statistically significant difference compared to Placebo+Placebo $(p<0.05),{ }^{* *}$ indicates a statistically significant difference compared to Placebo+Placebo $(p<0.01),{ }^{* * *}$ indicates a statistically significant difference compared to Placebo+Placebo $(p<0.001)$ 


\section{Change in time until exhaustion}

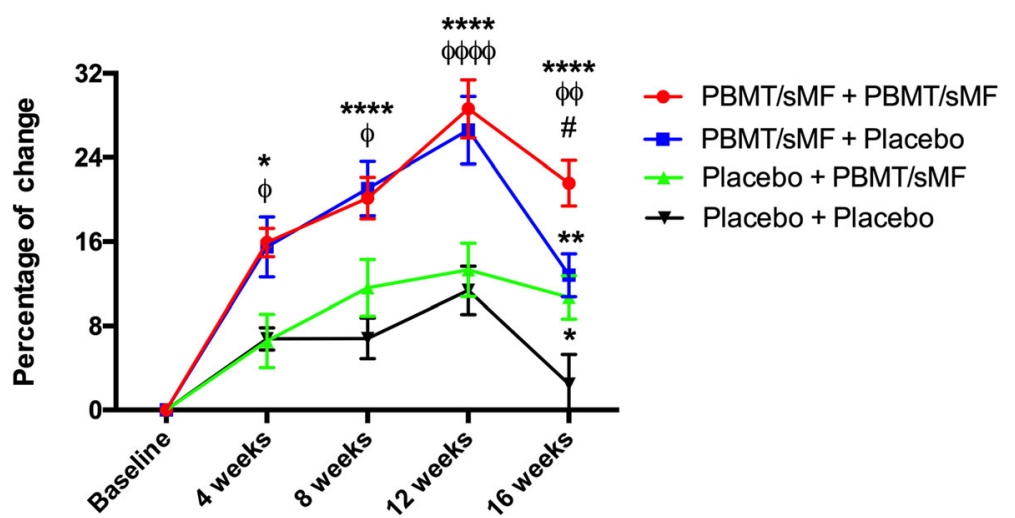

Fig. 3 Percentage of change in time to exhaustion. The data are presented in mean and SEM. * indicates statistical significance of $p<0.05$ compared to Placebo+Placebo; ** indicates statistical significance of $p<0.01$ compared to Placebo+Placebo; **** indicates statistical significance of $p<0.0001$ compared to Placebo+Placebo; ${ }^{\varnothing}$ indicates statistical significance of $p<0.05$ compared to Placebo+PBMT/sMF; ${ }^{\varnothing \varnothing}$ indicates statistical significance of $p<0.01$ compared to Placebo+PBMT/sMF; ${ }^{ø \varpi \varpi}$ indicates statistical significance of $p<0.0001$ compared to Placebo+PBMT/sMF; and \# indicates statistical significance of $p<0.05$ compared to PBMT/sMF + Placebo

difference $(p<0.01$ and $p<0.05$, respectively) compared to the Placebo+Placebo group at the 16th week.

Figure 5 shows the percentage of change in body fat percentage throughout the study. Only the group treated with PBMT/sMF throughout the whole study (PBMT/sMF + PBMT/sMF) demonstrated a percentage of change statistically superior $(p<0.05)$ in body fat mass compared to the Placebo+Placebo group, at the 16 th week. There were no significant differences in the other experimental groups or time-points tested.

\section{Discussion}

It is important to highlight that this was the first study to evaluate the effects of PBMT and sMF (PBMT/sMF) in the detraining period after aerobic training. In the present study, the application of PBMT/sMF before and after each training session, 3 times a week for 12 weeks, led to a statistically significant increase in the absolute values of $\mathrm{VO}_{2} \mathrm{max}$ at all evaluated time-points for the $\mathrm{PBMT} / \mathrm{sMF}+\mathrm{PBMT} / \mathrm{sMF}$ group and the PBMT/sMF + Placebo group compared to the Placebo+Placebo group.

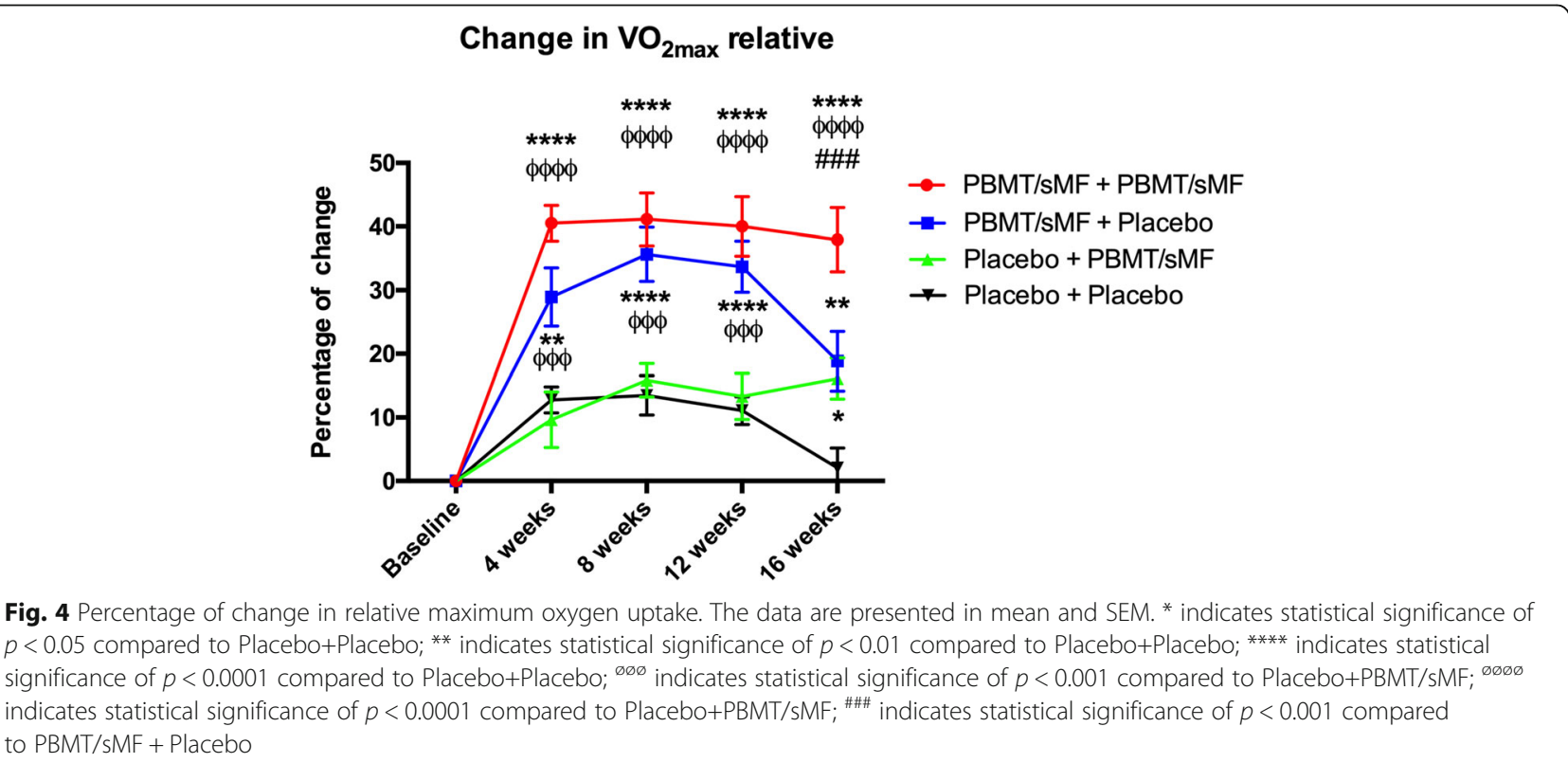




\section{Change in fat percentage}

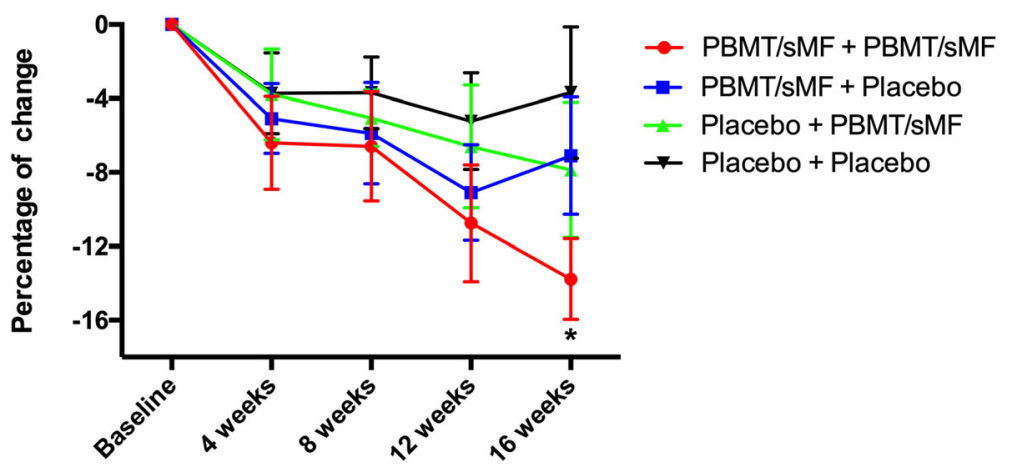

Fig. 5 Percentage of change in body fat. The data are presented in mean and SEM. * indicates statistical significance of $p<0.05$ compared to Placebo+Placebo

These positive results corroborate previous findings by our research group [25] as well as those reported by Paolillo et al. [34, 35]. Moreover, we observed that in the final training phase (12th week), PBMT/sMF improved the percentage of change of $\mathrm{VO}_{2} \max$ in $40.02 \%$ and time until exhaustion in $28.62 \%$. Again, our results further validate the findings of Miranda et al. [25] and Paolillo et al. $[25,34,35]$, thereby increasing the body of evidence supporting the benefits of PBMT in association with aerobic training.

As previously mentioned, the main goal of this study was to investigate the effects of PBMT/sMF during a 4week period without physical activity. At the end of this period, the group that received $\mathrm{PBMT} / \mathrm{sMF}$ throughout the whole study (PBMT/sMF + PBMT/sMF group) showed a statistically significant difference in the absolute values $(p<0.01)$ of $\mathrm{VO}_{2}$ max compared to the Placebo+Placebo group. Interestingly, $\mathrm{PBMT} / \mathrm{sMF}$ applied only during the training phase (PBMT/sMF + Placebo) also led to a statistically significant increase $(p<0.05)$ in the absolute values of $\mathrm{VO}_{2}$ max compared to the absolute values observed in the Placebo+Placebo group in the 16th week.

Previous studies have shown that a period of physical inactivity, specifically between 2 to 4 weeks [8], can lead to significant losses in aerobic capacity $[5,8]$. In our study, we also observed a decrease in percentage of change of $\mathrm{VO}_{2} \mathrm{max}$ in the Placebo+Placebo and PBMT/ sMF + Placebo groups during the detraining period (16th week) compared to the training period (12th week). Despite the lack of consensus regarding the magnitude of the loss of aerobic capacity provoked by a period of no physical activity, we believe that decreases in percentage of change of $\mathrm{VO}_{2}$ max observed in the Placebo+Placebo and $\mathrm{PBMT} / \mathrm{sMF}+$ Placebo groups are mainly related to the initial level of physical fitness of the practitioners as well as the duration of the detraining period, as described in the literature [8].

Similarly, the percentage of change in time until exhaustion was significantly higher in the PBMT/sMF + $\mathrm{PBMT} / \mathrm{sMF}$ group than in the Placebo+Placebo group $(21.55 \%$ versus $2.46 \%)$ in the 16 th week. Moreover, the Placebo+PBMT/sMF group showed better results than the Placebo+Placebo group (10.7\% versus $2.46 \%)$ in the 16th week. The group that received PBMT/sMF throughout the entire training and detraining periods also showed an increased change in percentage of fat. However, this same group showed a statistically significant decrease in this percentage, in relation to the Placebo+Placebo group, only in the detraining period (16th week). These findings allow us to infer that the application of PBMT/sMF throughout the whole study aided in reducing the body fat of the participants, even during a period without training. Similar findings were previously presented with the use of PBMT/sMF applied before and after aerobic training sessions [25]. These findings reiterate the increased effectiveness of PBMT/sMF when applied in both training and detraining periods.

The positive results obtained from the use of PBMT/ sMF can be attributed to its ergogenic effects on aerobic training, similar to the findings of Miranda et al. [25]. Moreover, the parameters used for irradiation in the present study are in line with those recently recommended by Leal-Junior et al. [10]. The establishment of optimised parameters is paramount for the effectiveness of PBMT/sMF since, in addition to the dose, the moment to perform the treatment and application sites should also be considered for therapy optimisation [36]. Given the importance of these factors, the protocol for irradiation followed the same parameters as those previously tested 
by Miranda et al. [25], which showed positive effects for the percentage of change in $\mathrm{VO}_{2} \max$, time until exhaustion, and body fat.

We believe that our findings are of great importance, especially in both rehabilitation and sports scenarios. It is well-known how difficult it is for elite athletes to maintain their performance levels, especially due to frequent injuries and consequent removal from their training routine. In this regard, our findings suggest that $\mathrm{PBMT} / \mathrm{sMF}$ can be an efficient alternative to physical trainers, athletes, and coaches in periods where aerobic training is interrupted.

Our findings may also bring up the question of whether PBMT/sMF is an acceptable technique for training and detraining in competitive and professional sports. The current version of the World Anti-Doping Code, published by the World Anti-Doping Agency (WADA) in 2015 [37], states that a substance or method can be considered as doping if two of the following three criteria are fulfilled: ' 1 - Medical or other scientific evidence, pharmacological effect or experience that the substance or method, alone or in combination with other substances or methods, has the potential to enhance or enhances sport performance; 2 - Medical or other scientific evidence, pharmacological effect or experience that the use of the substance or method represents an actual or potential health risk to the athlete; and 3 - WADA's determination that the use of the substance or method violates the spirit of sport described in the introduction to the code'. Furthermore, WADA also states that 'A substance or method shall also be included on the Prohibited List if WADA determines there is medical or other scientific evidence, pharmacological effect or experience that the substance or method has the potential to mask the Use of other Prohibited Substances or Prohibited Methods'.

However, since, as far as we know, PBMT/sMF does not have side effects or potential health risks, it is likely not fulfilling criterion number 2. Moreover, as the last criterion is more political than scientific, the decision about whether PBMT/sMF use should be included in the prohibited list of WADA is for the members of the executive board committee to decide.

A limitation of our study is that we did not investigate the mechanisms of action behind the positive effects presented by PBMT/sMF, which limits us to provide mechanistic insights related to the ergogenic effects of the therapy. Despite the wide number of reports regarding the modulatory effects of $\mathrm{PBMT} / \mathrm{sMF}$ in cellular metabolism [11, 12, 23] and on the partial pressure of oxygen and oxygen saturation in peripheral blood cells [26], we believe that further research should focus on the mechanisms of action behind the ergogenic effects of PBMT/sMF in humans. A second limitation of this study is that we did not monitor the habitual activity of our participants during detraining, which should also be considered in further research. The last limitation of this study is that only 4 weeks of detraining after 12 weeks of aerobic endurance training were analysed; however, the attenuation of the losses due to the interruption of physical activity is of great value, both for professional and amateur athletes. These benefits can also be transferred to several different scenarios, such as individuals affected by illness and those who are bedridden or unable to perform physical exercises for rehabilitation.

\section{Conclusions}

In the present study, the use of PBMT/sMF was able to potentiate the effects of aerobic training and reduce losses caused by the detraining period. Therefore, we believe that the results of the study are relevant and suggest a new perspective on the use of PBMT/sMF by recognising its effectiveness in maintaining the benefits obtained after aerobic training, during the detraining period.

\section{Abbreviations \\ PBMT: Photobiomodulation therapy; LEDs: Light-emitting diodes; sMF: Static magnetic field; $\mathrm{VO}_{2}$ max: Maximum oxygen consumption; $\mathrm{CK}$ : Creatine kinase;} $\mathrm{PO}_{2}$ : Partial pressure of oxygen; $\mathrm{SaO}_{2}$ : Oxygen saturation

\section{Acknowledgements \\ Not applicable.}

\section{Authors' contributions}

PRVP, HLC, and ECPL-J contributed to the concept and design of the study, established the hypothesis, and wrote the original proposal. PRVP, HLC, SST, CSMM, NFR, ALP, MFDO, MNSA, MCS, IETT, EFM, PTCC, and ECPL-J contributed significantly to manuscript creation. SST and PTCC performed critical revisions of the manuscript. PRVP, HLC, and ECPL-J wrote the final version of the manuscript. All authors have read and approved the final version of the manuscript.

\section{Funding}

This study is supported by the São Paulo Research Foundation - FAPESP through PhD scholarship number 2016/11878-5 granted to Paulo Roberto Vicente de Paiva and by the Brazilian Council of Science and Technology Development - CNPq grant number 310281/2017-2 granted to Professor Ernesto Cesar Pinto Leal-Junior.

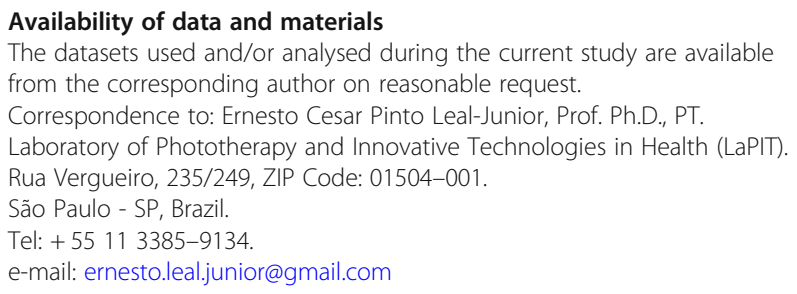

Ethics approval and consent to participate

The study followed the ethical guidelines of and was approved by the Research Ethics Committee of Nove de Julho University (protocol number 1781602). 


\section{Competing interests}

Professor Ernesto Cesar Pinto Leal-Junior receives research support from Multi Radiance Medical (Solon - OH, USA), a therapeutic device manufacturer. The remaining authors declare that they have no conflict of interests.

\section{Author details}

'Laboratory of Phototherapy and Innovative Technologies in Health (LaPIT), Nove de Julho University, Rua Vergueiro, 235/249, São Paulo, SP 01504-001, Brazil. ${ }^{2}$ Postgraduate Program in Rehabilitation Sciences, Nove de Julho University, São Paulo, SP, Brazil. ${ }^{3}$ Physiotherapy Research Group, Department of Global Public Health and Primary Care, University of Bergen, Bergen, Norway. ${ }^{4}$ ELJ Consultancy, Scientific Consultants, São Paulo, SP, Brazil.

Received: 18 September 2019 Accepted: 24 March 2020 Published online: 10 April 2020

\section{References}

1. Warburton DE, Nicol CW, Bredin SS. Health benefits of physical activity: the evidence. CMAJ. 2006;174:801-9.

2. Warburton DE, Katzmarzyk PT, Rhodes RE, Shephard RJ. Evidence-informed physical activity guidelines for Canadian adults. Can J Public Health. 2007;98: S16-68.

3. Garber CE, Blissmer B, Deschenes MR, et al. Quantity and quality of exercise for developing and maintaining cardiorespiratory, musculoskeletal, and Neuromotor fitness in apparently healthy adults. Med Sci Sports Exerc. 2011; 43(7):1334-59.

4. Hatle H, Støbakk PK, Mølmen HE, et al. Effect of 24 sessions of high-intensity aerobic interval training carried out at either high or moderate frequency, a randomized trial. PLoS One. 2014;9(2):e88375.

5. Lovell DI, Cuneo R, Wallace J, McLellan C. The hormonal response of older men to sub-maximum aerobic exercise: the effect of training and detraining. Steroids. 2012;77(5):413-8.

6. Libardi CA, Souza GV, Cavaglieri CR, et al. Effect of resistance, endurance, and concurrent training on TNF-alfa, IL-6, and CRP. Med Sci Sports Exerc. 2012:44(1):50-6.

7. Willis $L H$, Slentz CA, Bateman LA, et al. Effects of aerobic and/or resistance training on body mass and fat mass in overweight or obese adults. J Appl Physiol. 2012;113:1831-7.

8. Sousa AC, Neiva HP, Izquierdo M, Cadore EL, Alves AR, Marinho DA Concurrent training and detraining: brief review on the effect of exercise intensities. Int J Sports Med. 2019:40:747-55.

9. Stebbings GK, Morse $\mathrm{Cl}$, McMahon GE, Onambele GL. Resting arteria diameter and blood flow changes with resistance training and detraining in healthy young individuals. J Athl Train. 2013;48:209-19.

10. Leal-Junior ECP, Lopes-Martins RAB, Bjordal JM, et al. Clinical and scientific recommendations for the use of photobiomodulation therapy in exercise performance enhancement and post-exercise recovery: current evidence and future directions. Braz J Phys Ther. 2019:23:71-5.

11. Hayworth CR, Rojas JC, Padilla E, et al. In vivo low-level light therapy increases cytochrome oxidase in skeletal muscle. Photochem Photobiol. 2010;86:673-80.

12. Albuquerque-Pontes GM, Vieira RP, Tomazoni SS, et al. Effect of preirradiation with different doses, wavelengths, and application intervals of low level laser therapy on cytochrome c oxidase activity in intact skeletal muscle of rats. Lasers Med Sci. 2015;30:59-66.

13. Leal Junior EC, Lopes-Martins RA, Baroni BM, et al. Effect of $830 \mathrm{~nm}$ lowlevel laser therapy applied before high-intensity exercises on skeletal muscle recovery in athletes. Lasers Med Sci. 2009;24:857-63.

14. Antonialli FC, De Marchi T, Tomazoni SS, et al. Phototherapy in skeletal muscle performance and recovery after exercise: effect of combination of superpulsed laser and light-emitting diodes. Lasers Med Sci. 2014;29:1967-76.

15. de Paiva PR, Tomazoni SS, Johnson DS, et al. Photobiomodulation therapy (PBMT) and/or cryotherapy in skeletal muscle restitution, what is better? A randomized, double-blinded, placebo-controlled clinical trial. Lasers Med Sci. 2016:31:1925-33.

16. Leal Junior EC, Lopes-Martins RA, Rossi RP, et al. Effect of cluster multi-diode light emitting diode therapy (LEDT) on exercise-induced skeletal muscle fatigue and skeletal muscle recovery in humans. Lasers Surg Med. 2009;41:572-7.

17. Leal ECP, Lopes-Martins RAB, Frigo L, et al. Effects of low-level laser therapy (LLLT) in the development of exercise-induced skeletal muscle fatigue and changes in biochemical markers related to postexercise recovery. J Orthop Sports Phys Ther. 2010;40:524-32.
18. Pinto HD, Vanin AA, Miranda EF, et al. Photobiomodulation therapy improves performance and accelerates recovery of high-level Rugby players in field test: a randomized, crossover, double-blind, placebo-controlled clinical study. J Strength Cond Res. 2016;30:3329-38.

19. World Health Organization. "Environmental Health Criteria 232," in Static fields. Geneva: World Health Organization; 2006.

20. Okano $\mathrm{H}$. Effects of static magnetic fields in biology: role of free radicals. Front Biosci. 2008;13:6106-25.

21. Coballase-Urrutia E, Navarro L, Ortiz UL, et al. Static magnetic fields modulate the response of different oxidative stress markers in a restraint stress model animal. Biomed Res Int. 2018;2018:3960408.

22. Wang D, Wang Z, Zhang $L$, et al. Cellular ATP levels are affected by moderate and strong static magnetic fields. Bioelectromagnetics. 2018;39(5):352-60.

23. Friedmann H, Lipovsky A, Nitzan Y, Lubart R. Combined magnetic and pulsed laser fields produce synergistic acceleration of cellular electron transfer. Laser Ther. 2009;18:137-41.

24. Vanin AA, De Marchi T, Tomazoni SS, et al. Pre-exercise infrared low-level laser therapy $(810 \mathrm{~nm})$ in skeletal muscle performance and post exercise recovery in humans, what is the optimal dose? A randomized, double-blind, placebo-controlled clinical trial. Photomed Laser Surg. 2016;34:473-82.

25. Miranda EF, Tomazoni SS, de Paiva PRV, et al. When is the best moment to apply photobiomodulation therapy (PBMT) when associated to a treadmill endurance-training program? A randomized, triple-blinded, placebocontrolled clinical trial. Lasers Med Sci. 2018:33:719-27.

26. Wasik M, Gorska E, Modzelewska M, et al. The influence of low-power helium-neon laser irradiation on function of selected peripheral blood cells. J Physiol Pharmacol. 2007:58(5):729-37.

27. de Paiva PRV, Casalechi HL, Tomazoni SS, et al. Effects of photobiomodulation therapy in aerobic endurance training and detraining in humans: protocol for a randomized placebo-controlled trial. Medicine. 2019:98:e15317.

28. Grandinétti VS, Miranda EF, Johnson DS, et al. The thermal impact of phototherapy with concurrent super-pulsed lasers and red and infrared LEDs on human skin. Lasers Med Sci. 2015;30:1575-81.

29. Keteyian SJ, Brawner CA, Ehrman JK, et al. Reproducibility of peak oxygen uptake and other cardiopulmonary exercise parameters: implications for clinical trials and clinical practice. Chest. 2010;138(4):950-5.

30. De Marchi T, Leal-Junior EC, Bortoli C, et al. Low-level laser therapy (LLLT) in human progressive-intensity running: effects on exercise performance, skeletal muscle status, and oxidative stress. Lasers Med Sci. 2012;27:231-6.

31. Miranda EF, Vanin AA, Tomazoni SS, et al. Using pre-exercise photobiomodulation therapy combining super-pulsed lasers and lightemitting diodes to improve performance in progressive cardiopulmonary exercise tests. J Athl Train. 2016:51:129-35.

32. Tomazoni SS, Machado CDSM, De Marchi T, et al. Infrared low-level laser therapy (photobiomodulation therapy) before intense progressive running test of high-level soccer players: effects on functional, muscle damage, inflammatory, and oxidative stress markers-a randomized controlled trial. Oxidative Med Cell Longev. 2019;2019:6239058.

33. Marfell-Jones M, Olds T, Stewart A, Carter L. International standards for anthropometric assessment. ISAK, Potchefstroom (South Africa); 2006.

34. Paolillo FR, Milan JC, Aniceto IV, et al. Effects of infrared-LED illumination applied during high-intensity treadmill training in postmenopausal women. Photomed Laser Surg. 2011;29:639-45.

35. Paolillo FR, Corazza AV, Borghi-Silva A, et al. Infrared LED irradiation applied during high-intensity treadmill training improves maximal exercise tolerance in postmenopausal women: a 6-month longitudinal study. Lasers Med Sci. 2013;28: 415-22.

36. Vanin AA, Verhagen E, Barboza SD, Costa LOP, Leal-Junior ECP. Photobiomodulation therapy for the improvement of muscular performance and reduction of muscular fatigue associated with exercise in healthy people: a systematic review and meta-analysis. Lasers Med Sci. 2018, 33:181-214.

37. World Anti-Doping Agency - WADA (2015): World anti-doping code. Available at: https://www.wada-ama.org/en/resources/the-code/world-antidoping-code. Accessed 14 Feb 2020.

\section{Publisher's Note}

Springer Nature remains neutral with regard to jurisdictional claims in published maps and institutional affiliations. 